\title{
CONVEX HYPERSURFACES WITH PINCHED SECOND FUNDAMENTAL FORM
}

\author{
RICHARD S. HAMILTON
}

1.

In this paper we prove the following result.

1.1. Main Theorem. Let $M^{n}$ be a smooth strictly convex complete hypersurface bounding a region in $\mathbb{R}^{n+1}$. Suppose that its second fundamental form is $\varepsilon$-pinched, in the sense that

$$
H_{i j} \geq \varepsilon H g_{i j}
$$

where $g_{i j}$ is the induced Riemannian metric, $H_{i j}$ the second fundamental form, and its trace $H$ is the mean curvature, for some $\varepsilon>0$. Then $M^{n}$ is compact.

This result can be used to simplify the proof of Huisken's theorem [Hu1] for the mean curvature flow of a convex hypersurface in Euclidean space. We would especially like to thank Mike Gage, who pointed out the relation of the pinching condition to quasi-conformal maps, and Burt Rodin, who showed us the basic estimates for quasi-conformal maps.

2.

Suppose now that $M^{n} \subseteq \mathbb{R}^{n+1}=\mathbb{R}^{n} \times \mathbb{R}^{1}$ is written as the graph over a convex open set $U \subseteq \mathbb{R}^{n}$ of a strictly convex function

$$
y=f\left(x_{1}, \ldots, x_{n}\right)
$$

such that $y \rightarrow+\infty$ as $X=\left(x_{1}, \ldots, x_{n}\right)$ approaches the boundary of $U$. By translating upward if necessary, since $y$ is bounded below we can assume $y \geq e$ 
everywhere, so that $\ell n \ell n y \geq 0$. Let

$$
d s^{2}=g_{i j} d x^{i} d x^{j}
$$

be the Riemannian metric induced on $M$, so that

$$
g_{i j}=I_{i j}+\frac{\partial y}{\partial x^{i}} \frac{\partial y}{\partial x^{j}} .
$$

The following observation is fundamental to the proof.

Theorem 2.1. The conformally equivalent metric

$$
d \tilde{s}=\frac{d s}{y \ln y}
$$

is complete with finite volume.

Proof. First we show $d \tilde{s}$ is complete. For any path going to $\infty$ we have $y \rightarrow$ $+\infty$ and $d s \geq d y$. Therefore its length $L$ satisfies

$$
\left.L \geq \int_{b}^{\infty} \frac{d y}{y \ell n y}=\ln \ell n y\right]_{b}^{\infty}=\infty
$$

which is what we desire.

To estimate the volume, we observe that, because $y$ is a strictly convex function of $x$, outside of a compact set we must have

$$
\left|\frac{\partial y}{\partial x^{i}}\right| \geq \delta
$$

for some $\delta>0$. Let $d V$ denote the volume element on $M$ in the induced metric $d s$, which in $X$ coordinates is

$$
d V=\sqrt{\operatorname{det}\left(I_{i j}+\frac{\partial y}{\partial x^{i}} \frac{\partial y}{\partial x^{j}}\right)} d x^{1} d x^{2} \cdots d x^{n}
$$

and let $d V_{y}$ denote the volume element of the part of $M$ between height $y$ and height $y+d y$. We can divide $M$ into $n$ pieces where $\partial y / \partial x^{1}$ is largest, or $\partial y / \partial x^{2}$, or so on, and estimate $d V_{y}$ from above on each piece. Where $\partial y / \partial x^{1}$ is largest, we take $y, x^{2}, \ldots, x^{n}$ as coordinates. Then

$$
\sqrt{\operatorname{det}\left(I_{i j}+\frac{\partial y}{\partial x^{i}} \frac{\partial y}{\partial x^{j}}\right)} \leq C\left|\frac{\partial y}{\partial x^{1}}\right|
$$


since $\partial y / \partial x^{1}$ is larger than the other derivatives, and $\left|\partial y / \partial x^{i}\right| \geq \delta>0$. This makes

$$
d V \leq C d y d x^{2} \cdots d x^{n}
$$

on this patch. Moreover our gradient estimate shows that

$$
|X| \leq C y
$$

for a suitably large constant. Therefore

$$
d V_{y} \leq C y^{n-1} d y
$$

since the region of integration over $x^{2}, \ldots, x^{n}$ to get $d V_{y}$ from $d V$ has its volume bounded by $C y^{n-1}$. Then

$$
d \widetilde{V}_{y} \leq \frac{C}{y \ln ^{n} y} d y \quad \text { and } \quad \widetilde{V} \leq C \int \frac{d y}{y \ln ^{n} y}<\infty
$$

3.

By contrast we have the following result.

Theorem 3.1. Let $U$ be an open subset of the unit sphere $S^{n}$ which is not empty and whose closure is not the whole sphere. Then there is no metric on $U$ conformal to the round metric which is complete with finite volume.

Proof. By hypothesis we can find some point $N$ which is contained in $U$, and some point $S$ which avoids the closure $U$. By a conformal transformation, we can take $N$ to be the north pole and $S$ the south pole. We can then find an $\varepsilon>0$ so that the $\varepsilon$-ball around $N$ lies in $U$, while the $\varepsilon$-ball around $S$ avoids $U$. We can then find a conformal map of the sphere $S^{n}$ minus these two balls to the cylinder $S^{n-1} \times[0, L]$, taking the boundary of the $\varepsilon$-ball around $N$ to $S^{n-1} \times\{0\}$ and the boundary of the $\varepsilon$-ball around $S$ to $S^{n-1} \times\{L\}$. The part of $U$ outside the $\varepsilon$-ball around $N$ will map to some relatively open subset $W$ of the cylinder which contains $S^{n-1} \times\{0\}$ and avoids $S^{n-1} \times\{L\}$. The subset $W$ will be a non-compact manifold with one compact boundary component $S^{n-1}$. Any complete metric on $U$ wuth finite volume conformal to the round metric on $S^{n}$ would give a complete metric with finite volume on $W$ conformal to the product metric on $S^{n-1} \times[0, L]$. We show that such cannot exist. 
We introduce coordinates $\Theta=\left(\theta_{1}, \ldots, \theta_{n-1}\right)$ on $S^{n-1}$ and $t$ on $[0, L]$. Let $d \sigma$ denote the metric on $S^{n-1}$ and $d \mu$ the volume form. Then $d s^{2}=d \sigma^{2}+d t^{2}$ is the product metric on $S^{n-1} \times[0, L]$, and $d V=d \mu d t$ is the product volume form. For every $\Theta \in S^{n-1}$, there will be a first point $t=h(\Theta)$ where the pair is no longer in $W$, Of course $h$ may not be a continuous function, and the pair may re-enter $W$ for larger values of $t$. This does not matter. Any conformally equivalent metric on $W$ is given by

$$
d \tilde{s}=\rho(\Theta, t) d s
$$

for some function $\rho$ defined at least for $0 \leq t<h(\Theta)$. The corresponding volume form is

$$
d \widetilde{V}=\rho^{n} d \mu d t .
$$

If the total volume $\tilde{V}$ of the conformally equivalent metric is finite, we have

$$
\iint_{W} \rho^{n} d \mu d t=\widetilde{V}<\infty
$$

By Hölder's inequality

$$
\iint_{W} \rho d \mu d t \leq\left\{\iint_{W} \rho^{n} d \mu d t\right\}^{1 / n}\left\{\iint_{W} 1 d \mu d t\right\}^{\frac{n-1}{n}}
$$

and surely

$$
\iint_{W} 1 d \mu d t \leq L \operatorname{vol}\left(S^{n-1}\right)<\infty
$$

Therefore

$$
\iint_{0 \leq t<h(\Theta)} \rho(\Theta, t) d \mu d t<\infty
$$

On the other hand, if we integrate first in $t$, we see that

$$
\int_{S^{n-1}}\left\{\int_{0}^{h(\Theta)} \rho(\Theta, t) d t\right\} d \mu \geq \operatorname{vol}\left(S^{n-1}\right) \inf _{\Theta} \int_{0}^{h(\Theta)} \rho(\Theta, t) d t
$$

and therefore

$$
\inf _{\Theta} \int_{0}^{h(\Theta)} \rho(\Theta, t) d t<\infty .
$$

But along a path where $\Theta$ is constant we have $d \tilde{s}=\rho d t$. Thus there is some $\Theta$ where the path from $(\Theta, 0)$ to $(\Theta, h(\Theta))$ has finite length. This shows that the metric is not complete, and proves Theorem 3.1. 
4.

It only remains to observe that the Gauss map gives a diffeomorphism of the convex hypersurface $M^{n}$ onto an open subset $U$ of the sphere $S^{n}$ which lies in a hemisphere. Thus $U$ is not empty and its closure is not all of $S^{n}$. However, the pinching condition

$$
H_{i j} \geq \varepsilon H g_{i j}
$$

is equivalent to the assertion that the Gauss map is quasi-conformal. Since $M$ has a conformally equivalent metric which is complete with finite volume, so must $U$. But this is impossible.

5.

Finally we indicate how to use this result to prove Huisken's result [Hu1] on compact convex hypersurfaces shrinking to a point under the Mean Curvature flow. Huisken shows that the pinching estimate

$$
H_{i j} \geq \varepsilon H g_{i j}
$$

is preserved by the flow. This is an easy consequence of the maximum principle. The usual derivative estimates guarantee that after a short time we can control all the covariant derivatives of the $H_{i j}$ in terms of the size of $H_{i j}$ itself, which in turn is controlled by $H$ alone when $H_{i j}>0$. As a result, the solution surely exists until $H$ is unbounded as $t \rightarrow T$ for some $T<\infty$.

Moreover a standard argument allows us to "blow up" the singularity by taking a limit of a sequence of translations and dilations of solutions. We distinguish two cases. In Case I, where we have

$$
\lim \sup (T-t) H^{2}=A<\infty
$$

the limit will be a solution to the Mean Curvature flow on $-\infty<t<A$ with $H=1$ at the origin at time 0 and

$$
0<H \leq \frac{A}{A-t}
$$

everywhere for all time. Because of the pinching condition our result shows the limit must be compact, and then Huisken's uniqueness result [Hu2] shows 
it must be the round sphere as desired. In Case II, where we have

$$
\lim \sup (T-t) H^{2}=\infty
$$

the limit will be a solution to the Mean Curvature flow on $-\infty<t<\infty$ with $H=1$ at the origin at time 0 and

$$
0<H \leq 1
$$

everywhere for all time. The Harnack estimate applied with the strong maximum principle [Ha] shows this limit must be a translating soliton. All we need observe, however, is that it lasts forever and hence cannot be compact. Then the pinching estimate gives a contradiction, so this case is ruled out.

\section{REFERENCES}

[Ha] Hamilton, R. S., The Harnack estimate for the mean curvature flow, to appear in J. Diff. Geom.

[Hu1] Huisken, G., Flow by mean curvature of convex hypersurfaces into spheres, J. Diff. Geom. 20 (1984), 237-266.

[Hu2] Huisken, G., Asymptotic behavior for singularities of the mean curvature flow, J. Diff. Geom. 31 (1990), 285-299.

University of California, SAN Diego, U. S. A.

RECEIVED OCTOBER 7, 1993 